\title{
Significantly Earlier Ambulation and Reduced Risk of Near-Falls with Continuous Infusion Nerve Blocks: A Retrospective Pilot Study of Adductor Canal Block Compared to Femoral Nerve Block in Total Knee Arthroplasty
}

\section{Yutaka Fujita}

Uonuma Institute of Community Medicine, Niigata University Medical and Dental Hospital, Uonuma Kikan Hospital

\section{Hisashi Mera ( $\nabla$ hisme0214@gmail.com )}

Uonuma Institute of Community Medicine, Niigata University Medical and Dental Hospital, Uonuma Kikan Hospital

\section{Tatsunori Watanabe}

Uonuma Institute of Community Medicine, Niigata University Medical and Dental Hospital, Uonuma Kikan Hospital

Kenta Furutani

Niigata University Medical and Dental Hospital, Uonuma Kikan Hospital

Haruna O. Kondo

Uonuma Institute of Community Medicine, Niigata University Medical and Dental Hospital, Uonuma Kikan Hospital

\section{Takao Wakai}

Uonuma Institute of Community Medicine, Niigata University Medical and Dental Hospital, Uonuma Kikan Hospital

\section{Hiroyuki Kawashima}

Niigata University Graduate School of Medical and Dental Sciences

\section{Akira Ogose}

Uonuma Institute of Community Medicine, Niigata University Medical and Dental Hospital, Uonuma Kikan Hospital

\section{Research Article}

Keywords: Total knee arthroplasty, Adductor canal block, early ambulation recovery

Posted Date: November 30th, 2021 
DOI: https://doi.org/10.21203/rs.3.rs-1119118/v1

License: (c) (1) This work is licensed under a Creative Commons Attribution 4.0 International License. Read Full License 


\section{Abstract}

Background: One option in total knee arthroplasty (TKA) perioperative pain management is femoral nerve block (FNB). Its association with quadriceps weakness has led to a focus on adductor canal block (ACB), with the aim of avoiding weakness in adjacent muscles. This study reviewed cases at our institution.

Methods: In this pilot non-blinded study, nerve block (FNB or ACB) was performed under ultrasonic guidance after induction of general anesthesia, with an initial bolus followed by continuous levobupivacaine infusion into the perineurium as needed until postoperative day (POD) 2 or 3 . Pain levels and falls/near-falls with knee-buckling were monitored from POD 1 to POD 3 . The score on the manual muscle test, MMT ( 0 to 5,5 being normal), of the patients who had been able to ambulate on POD 1, was investigated.

Results: A total of 73 TKA cases, 37 FNB and 36 ACB, met the inclusion criteria. Episodes of near-falls in the form of knee-buckling were witnessed in 14 (38\%) cases in the FNB group and in $4(11 \%)$ in the ACB group ( $p=0.0068)$. In the ACB group, $81.1 \%$ of patients were able to ambulate with parallel bars on POD 1 , while only $44.4 \%$ of FNB patients could do so $(p=0.0019)$. The quadriceps MMT values of patients able to ambulate with parallel bars on POD 1 in the ACB group was 2.82 , significantly higher than 1.97 in the FNB group $(p=0.0035)$. There were no significant differences in pain as measured with a numerical rating scale (NRS) through POD 3.

Conclusion: Compared to FNB, ACB was associated with significantly less knee-buckling and earlier initiation of ambulation post-TKA, with better quadriceps muscle strength. These findings support the use of ACB as the anesthesia method of choice for TKA.

\section{Background}

Total knee arthroplasty (TKA) is performed to treat osteoarthritis (OA) and rheumatoid arthritis by relieving pain and restoring function. Pain management is important for prompt restoration of ambulation in the immediate postoperative period and for minimization of the risk of complications, such as deep vein thrombosis $[1,2]$.

Peripheral nerve blocks (PNB) are important in contemporary multimodality pain regimens aimed at reducing the potential side effects of other analgesics, such as opioids [3]. Complications of PNB include neurovascular injury, systemic toxicity, and, when using a femoral nerve block (FNB), weakness quadriceps muscle weakness [3]. Continuous PNB, in which an infusion catheter is inserted percutaneously around the peripheral nerve, can be expected to have a long-term therapeutic effect. Despite concern about local infection [4], the risk of severe long-term complications is rare, and catheters kept in place up to 76 hours after surgery have a low complication rate [3-5].

FNB has been widely used due to its ease of application and its excellent pain relief after TKA [6-12]. However, some studies have shown that FNB weakens quadriceps strength $[13,14]$, which may result in 
an increased risk of falls during rehabilitation [15-22]. FNB is focused on the proximal femoral nerve, which contains both motor and sensory fibers [23]. The motor blockade can cause quadriceps weakness, with the associated difficulties in establishing ambulation potentially slowing the rehabilitation process [24-26].

Recent findings have shown the potential of adductor canal block (ACB), which can provide equally effective pain relief without affecting quadriceps strength [27-29]. As the injection site is distal to that of the FNB, only the purely sensory saphenous nerve in the adductor canal is anesthetized, preserving quadriceps strength $[13,14,30]$. Studies have shown that ACB is an equally effective alternative to FNB, allowing earlier recovery of ambulation compared to FNB after TKA [24, 26, 27, 31-34]. Given preoperative morbidities such as lung disease, it may be unrealistic to expect all patients to ambulate on POD 1, and patients require close monitoring in the early postoperative period to prevent falls. Reports about the incidence of falls between FNB and ACB have been inconsistent, some due to differences in postoperative activity restriction $[14,21,25,35-39]$. Some studies have investigated the risk of postoperative falling, knee buckling/near falls or imbalance $[14,25,39]$.

We evaluated the differences in risk of knee-buckling/near-falls, the time to commencement of ambulation, and muscle strength between the two blocks. We analyzed TKA cases performed under general anesthesia at our institution, of which half received FNB and half received ACB, with continuous infusion out to POD 3. Demographics are shown in Table 1. We investigated the day of the commencement of ambulation, the risk of falling in the first three PODs, quadriceps muscle strength of the patients who had been able to commence ambulation with parallel bars on POD 1, and the effect of pain relief between ACB and FNB during that period.

\section{Methods}

After approval for this retrospective study was obtained from the Institutional Review Boards of the authors' affiliated institutions, we collected clinical data of patients who had undergone primary unilateral TKA under general anesthesia with FNB (October 2016 to September 2017) and ACB (October 2017 to March 2019) as part of routine anesthesia protocols. We excluded patients undergoing revision TKA and those with incomplete physical therapist notes during rehabilitation. The protocol for TKA patients at our institution uses a linear ultrasound transducer placed parallel to the groin to guide advancement of the needle to the femoral nerve (short axis) on the ventral side of the iliopsoas muscle (FNB) or the perineurium of the saphenous nerve in the adductor canal (ACB) to inject an initial bolus of $200 \mathrm{mg}$ of $0.25 \%$ levobupivacaine with $10 \mathrm{~mL}$ saline. A catheter is then inserted approximately $3 \mathrm{~cm}$ into the perineurium, and levobupivacaine and saline are infused continuously until POD 2 to 3 . Anesthesiologists well-experienced in these procedures performed the regional blocks for both FNB and ACB.

Patient charts were reviewed to determine demographics such as age, sex, body mass index (BMI), and comorbidities, including the incidence of dementia, cardiovascular diseases (congestive heart failure, ischemic heart disease including ischemic coronary artery disease with or without stenting, prior 
myocardial infarction, arrhythmias [including atrial fibrillation with pacemaker], and valvular disease), neurological disorders (prior cerebral infarction, cervical spinal myelopathy, and lumbar stenosis) without lower limb paralysis, pulmonary disorders (COPD, bronchial asthma, restrictive lung disease including interstitial pneumonia) without the need for home oxygen therapy, and diabetes mellitus (DM). Preoperative knee pain and function was assessed and scored using the Japanese Knee Osteoarthritis Measure (JKOM) clinical score, a patient-reported disease-specific assessment of knee OA which reflects the specificity of the Japanese cultural lifestyle [40] with reliability and validity comparable to the Medical Outcome Study 36-Item Short-Form Health Survey (SF-36) and the Western Ontario and McMaster Universities Arthritis Index (WOMAC) [40-42]. JKOM consists of 5 domains: JKOM-I for the evaluation of pain with a visual analogue scale (VAS: 0 to 100, 0 being normal). JKOM II to $V$ are for knee function components: JKOM-II has eight questions on knee pain and stiffness, JKOM-III has ten questions on activities of daily living, JKOM-IV has five questions on general activities that require leaving the house, and JKOM-V has two questions on the patient's perception of their overall health and the contribution made by knee symptoms [40]. The replies to JKOM II-V are scored on a five-point Likert scale, with 0 indicating "best" and 4 indicating "worst." To assess the patients' general condition on POD 1, we calculated total intraoperative blood loss, including the intra-articular suction drainage volume measured postoperatively from indwelling drains that were removed on POD 1. Most surgeries were conducted by a single arthroplasty surgeon and two senior residents under his supervision. All surgery in this study was performed using a pneumatic tourniquet during the procedure. A skin incision was made at the midline of the knee and a medial parapatellar approach was used to expose the joint [43] using a medial pivot prosthesis (Evolution ${ }^{\circledR}$ Medial-Pivot Knee System, Microport Orthopedics, Tokyo, Japan).

We reviewed physical therapist and nurse records to detect falls and near-falls with knee buckling (excluding falls from bed) during rehabilitation and hospitalization after TKA. Physical therapy (PT) commenced on the morning of POD 1, consisting of two 20-minute sessions per day, with all patients encouraged to bear weight and walk as soon as tolerable. The criteria for starting parallel bar ambulation training were knee stability and adequate pain control, i.e. PT assessment of knee stability during weight bearing including quadriceps muscle strength in the manual muscle test (MMT) (score, 0 to 5,5 being normal) [44], which could not be measured in all patients due to pain associated with knee motion [45, 46]. Patients unable to exert muscle strength or maintain a standing position due to pain [47] were not allowed to proceed to parallel bar ambulation training until they were able to stand. The day of commencement of ambulation was recorded for each patient (Table 2). We classified knee buckling as a near-fall, indicating insufficient muscle output leading to instability in standing and ambulation [39]. Falls and near-falls with knee-buckling from POD 1-POD 4 were documented by physical therapists or nurses. Unobserved ambulation was not allowed until patients had already been observed to walk with the parallel bars without problems. They were then allowed to use a walker or cane unobserved, usually on POD 4 or later. Along with assessment of mobility, we recorded the MMT scores of the quadriceps muscle in those patients who had been able to ambulate on POD 1.

The pain intensity of patients at rest was evaluated using a numerical rating scale (NRS-11). The nurses asked patients to rate their pain intensity three times daily. The mean values on POD 1, 2, and 3 were 
assessed.

Event frequencies of falls/near-falls with knee-buckling were analyzed using Fisher's exact test. Time to ambulation with parallel bars was compared using the Mann-Whitney U-test and Fisher's exact test. All continuous data, including age, BMI, blood loss, preoperative knee pain and function with the JKOM score, postoperative NRS, and MMT scores were compared using a two-sample, two-tailed Student's $t$ test. Some demographic data such as sex and comorbidities were compared using Fisher's exact test. A p value $<0.05$ was considered statistically significant. A power analysis is shown in the Supplementary Materials. All statistical analyses were performed with a modified version of R commander designed for specific use in biostatistics (EZR, Saitama Medical Center, Jichi Medical University, Saitama, Japan) [48].

\section{Results}

A total of 80 primary TKA cases were performed in our institution between October 2016 and March 2019. Three FNB cases were excluded due to the use of spinal anesthesia, and three other FNB cases were excluded because of unintentional removal of the infusion catheter within 24 hours after TKA. One case with ACB was excluded because cerebral infarction occurred on POD 1. The remaining 73 TKA cases met the inclusion criteria, for a total of 37 FNB and 36 ACB cases. Table 1 shows patient demographics; the ACB patients had a significantly higher mean BMI $(p=0.03)$ as well as greater blood loss $(355 \pm 212 \mathrm{~mL})$ than that the FNB patients $(271 \pm 128 \mathrm{~mL})$, albeit of borderline significance $(p=$ 0.045).

The records of the physical therapists and nurses showed no falls. Near-falls with knee-buckling occurred within PODs 1-3 (FNB: 14/37 [37.8\%], ACB: 4/36 [11.1\%], $p=0.0068$ ).

Statistically significant differences were observed between the groups in terms of initiation of ambulation in the immediate postoperative period (Table 2), with significantly more ACB patients ambulating on POD 1, at 1.27 (95\% Cl: 1.19-1.36) days, compared with 1.75 (95\% Cl: $1.47-2.03)$ days in the FNB group ( $p=$ 0.0019). MMT values for the quadriceps in patients who were able to ambulate with parallel bars on POD 1 in 30 cases in the ACB group was $2.82 \pm 0.88$ (95\% Cl: $2.49-3.14)$, significantly higher than the $1.97 \pm$ 0.87 (95\% Cl: 1.48-2.45) in 15 cases in the FNB group, except for one case in the FNB group who could not be evaluated due to pain $(p=0.0035)$.

There were no significant differences between the two groups in pain level on POD 1-3 (NRS of FNB and ACB; 3.8 and 3.6 [p = 0.6] on POD 1, 3.4 and 3.6 [p = 0.52] on POD 2, 3.2 and 3.0 [p = 0.64] on POD 3.

\section{Discussion}

No falls were noted during rehabilitation in either group. In contrast, the two groups differed significantly with regard to near-falls with knee-buckling (FNB, 14/37 [37.8\%]; ACB, 4/36 [11.1\%]; $p=0.0068)$.

Ambulation with parallel bars began significantly earlier in the ACB group compared with the FNB group. The mean MMT scores of the quadriceps of the patients who were able to ambulate with parallel bars on 
POD 1 were 2.82 (95\% Cl: 2.49-3.14) in the ACB group, significantly higher than the 1.97 (95\% Cl: $1.48-$ $2.45)$ in the FNB group. When evaluated by NRS, no statistically significant difference in pain relief between the two groups was seen.

Feibel et al. and Pelt et al. reported rates of falls after TKA with FNB continuous infusion of $0.7 \%$ (8 in 1190 cases) [16] and 2.7\% (19 in 707 cases) [21], respectively. Using continuous infusion, Bolarinwa et al. reported a fall rate of $0.13 \%$ ( 1 of 791 cases) with ACB compared to $1.3 \%$ (11 in 834 cases) with FNB, and recommended ACB as the preferred regional analgesia for the TKA procedure [38]. In our smaller study, the fall rate was $0 \%$ in both groups.

We defined knee-buckling as a near-fall since it can lead to falls, especially in the elderly [39]. Despite a significantly higher BMI in the ACB group, the frequency of knee-buckling was 14 patients $(37.8 \%)$ in the FNB group compared to $4(11.1 \%)$ in the ACB group $(p=0.0068)$. This number appears higher than that in the study by Thacher et al., which reported $17 / 129$ patients (13.2\%) in the FNB group and $3 / 150$ patients (2.0\%) in the ACB group [39]. This difference might be due to the older patient age at the time of surgery in our cases, differences in anesthesia administration (continuous infusion following bolus in our study vs. bolus alone in the previous report [39]), and possible differences in rehabilitation protocols. In particular, the difference between continuous catheter infusion and a single bolus administration may be significant. All of the near-falls with knee-buckling were confirmed by physical therapists, who monitored patients closely during the rehabilitation course. All knee-buckling episodes occurred within PODs 1-3, which may outline the minimum period when this close monitoring should be performed, although Thacher et al.'s study reported that 26 hours may be the window during which patients who receive a single administration of either FNB or ACB are most vulnerable [39]. Elkassaban et al. reported stopping the continuous infusion on the morning of POD 1 before starting physical therapy. Using the Tinelli fall risk scale $[25,49]$ and MMT values of the quadriceps, they found that the relative risk of fall with FNB was higher than that with ACB. In our study, patients received continuous infusion blocks, which were removed on POD 2 or 3 , widening the window. This suggests that careful supervision of all standing and ambulation, especially in FNB patients, is required until 24 hours after nerve blocks are removed to minimize fall risk.

A number of studies have suggested that ACB is associated with more prompt and better advancement of ambulation ability than FNB $[24,26,31,37]$. Shah et al. reported that the ambulation ability, the timed up-and-go (TUG), the ten-meter walk test, and the 30-second chair stand test [13] all had significantly better results with continuous ACB than continuous FNB anesthetic infusions (51.81s vs. $180.06 \mathrm{~s}$, 67.0s vs. 273.70 s, 5.25 vs. $1.52, p<0.001$, respectively) after removal of the catheter [31]. In our study, the quadriceps muscle strength of most patients did not recover sufficiently for them to perform unsupported tests, including the TUG, the ten-meter walk test, and the 30-second chair test, which Shah et al. did on POD 1 and 2.

In terms of quadriceps strength, although ACB is considered to be an almost pure sensory block, Jaeger et al., in a study of healthy volunteers, using a single bolus of $30 \mathrm{~mL}$ of $0.1 \%$ ropivacaine, reported that 
the mean reduction in quadriceps strength compared with $30 \mathrm{~mL}$ of saline in the opposite limb was $8 \%$ with ACB and $49 \%$ with FNB [13]. Kwofie et al., using single $15 \mathrm{~mL}$ bolus of $3 \%$ chloroprocaine, reported that isometric contraction of the quadriceps declined by $11 \% 60$ min after ACB block in one leg and $95 \%$ with FNB in the contralateral leg in healthy volunteers [14], which suggests that ACB does have a slight motor effect and still requires close attention from nursing staff during the early postoperative period.

In our retrospective study, we evaluated the intensity of postoperative pain in addition to the safety of ACB using NRS on POD 1, 2, and 3. Some studies have suggested that ACB is inferior to FNB with regard to the need for supplementary opioids [36, 39]. However, most studies, including those of Mudumbai et al. and Thacher et al. [36, 39], have supported the idea that ACB and FNB are equally effective in terms of pain relief $[24,35-37,39,50,51]$. Our study showed no statistically significant differences in NRS-11 scores between the two groups, indicating that ACB provided effective pain relief equivalent to that of FNB.

We demonstrated significant differences in mobility when walking with parallel bars and in the frequency of near-falls with knee-buckling between patients receiving continuous FNB vs. ACB, with earlier ambulation and fewer near-falls with knee-buckling in the ACB patients. The differences between the two groups may be due to the lower effect of ACB on quadriceps strength.

This study was limited by its retrospective design and small number of patients.

\section{Conclusion}

Attention has recently focused on multimodality analgesic regimens. Peripheral nerve blocks play an important role in joint surgery because of their efficacy and safety. Our study demonstrated that ACB provides better ambulation recovery with equivalent analgesia to FNB for TKA patients. Although many studies have compared FNB and ACB, few studies used continuous infusion, or investigated pain control, fall risk, and ambulation ability over POD 1-3. Our study shows the superior safety and efficacy of continuous ACB.

\section{Abbreviations}

TKA

total knee arthroplasty

$\mathrm{OA}$

osteoarthritis

PNB

peripheral nerve block

FNB

femoral nerve block

ACB 
adductor canal block

POD

post operative day

MMT

manual muscle test

PT

physical therapy

BMI

body mass index

COPD

chronic obstructive pulmonary disease

DM

diabetes mellitus

JKOM

Japanese knee osteoarthritis measure

VAS

visual analogue scale

NRS

numerical rating scale

$\mathrm{Cl}$

confidence interval

TUG

Time-Up-and-Go

\section{Declarations}

\section{Ethics approval and consent to participate}

This retrospective study has been approved by the Institutional Review Board of the authors' affiliated institutions (IRB\# 01-003). Prior to participation in the patient-reported survey, an opt-out method was used to guarantee patients and their families the opportunity to refuse participation. All components of the study were performed with their understanding and consent. If the patient preferred not to be a subject of the study, their medical information was immediately excluded from analysis and not used in the study.

\section{Availability of data and materials}

The data that support the findings of this study are available from the corresponding author, H.M., upon reasonable request. 


\section{Conflict of interest}

The authors declare that they have no conflicts of interest.

\section{Funding}

This study did not receive any funding.

\section{Authors' contributions}

Y.F., H.M. designed the research; Y.F., H.M., T.W., K.F., H.O.K., T.W. conducted the study; Y.F, H.M., H.O.K., T.W. acquired and analyzed the data; Y.F., H.M., H.O.K., T.W., H.K., A.O. interpreted the data; and Y.F., H.M. wrote the manuscript.

\section{Acknowledgments}

We thank Hironobu Ueshima MD, PhD for technical support for peripheral block, Mrs. Sachiko Hamazaki for supporting the collection of and inputting clinical data, and Libby Cone, MD, MA, from DMC Corp. (www.dmed.co.jp <http://www.dmed.co.jp/>) for editing drafts of this manuscript.

\section{References}

1. Ragucci MV, Leali A, Moroz A, Fetto J: Comprehensive deep venous thrombosis prevention strategy after total-knee arthroplasty. Am J Phys Med Rehabil 2003, 82(3):164-168.

2. Lei YT, Xie JW, Huang Q, Huang W, Pei FX: Benefits of early ambulation within $24 \mathrm{~h}$ after total knee arthroplasty: a multicenter retrospective cohort study in China. Mil Med Res 2021, 8(1):17.

3. Joshi G, Gandhi K, Shah N, Gadsden J, Corman SL: Peripheral nerve blocks in the management of postoperative pain: challenges and opportunities. J Clin Anesth 2016, 35:524-529.

4. Ilfeld BM: Continuous peripheral nerve blocks: a review of the published evidence. Anesth Analg 2011, 113(4):904-925.

5. Ilfeld BM, Mariano ER, Madison SJ, Loland VJ, Sandhu NS, Suresh PJ, Bishop ML, Kim TE, Donohue $\mathrm{MC}$, Kulidjian $\mathrm{AA}$ et al: Continuous femoral versus posterior lumbar plexus nerve blocks for analgesia after hip arthroplasty: a randomized, controlled study. Anesth Analg 2011, 113(4):897-903.

6. YaDeau JT, Cahill JB, Zawadsky MW, Sharrock NE, Bottner F, Morelli CM, Kahn RL, Sculco TP: The effects of femoral nerve blockade in conjunction with epidural analgesia after total knee 
arthroplasty. Anesth Analg 2005, 101(3):891-895.

7. Paul JE, Arya A, Hurlburt L, Cheng J, Thabane L, Tidy A, Murthy Y: Femoral nerve block improves analgesia outcomes after total knee arthroplasty: a meta-analysis of randomized controlled trials. Anesthesiology 2010, 113(5):1144-1162.

8. Hadzic A, Houle TT, Capdevila X, llfeld BM: Femoral nerve block for analgesia in patients having knee arthroplasty. Anesthesiology 2010, 113(5):1014-1015.

9. Albrecht E, Morfey D, Chan V, Gandhi R, Koshkin A, Chin KJ, Robinson S, Frascarolo P, Brull R: Singleinjection or continuous femoral nerve block for total knee arthroplasty? Clin Orthop Relat Res 2014, 472(5):1384-1393.

10. Beebe MJ, Allen R, Anderson MB, Swenson JD, Peters CL: Continuous femoral nerve block using $\mathbf{0 . 1 2 5 \%}$ bupivacaine does not prevent early ambulation after total knee arthroplasty. Clin Orthop Relat Res 2014, 472(5):1394-1399.

11. Chan EY, Fransen M, Parker DA, Assam PN, Chua N: Femoral nerve blocks for acute postoperative pain after knee replacement surgery. Cochrane Database Syst Rev 2014(5):CD009941.

12. Albrecht $E$, Guyen $O$, Jacot-Guillarmod $A$, Kirkham KR: The analgesic efficacy of local infiltration analgesia vs femoral nerve block after total knee arthroplasty: a systematic review and metaanalysis. Br J Anaesth 2016, 116(5):597-609.

13. Jæger P, Nielsen ZJ, Henningsen MH, Hilsted KL, Mathiesen O, Dahl JB: Adductor canal block versus femoral nerve block and quadriceps strength: a randomized, double-blind, placebo-controlled, crossover study in healthy volunteers. Anesthesiology 2013, 118(2):409-415.

14. Kwofie MK, Shastri UD, Gadsden JC, Sinha SK, Abrams JH, Xu D, Salviz EA: The effects of ultrasound-guided adductor canal block versus femoral nerve block on quadriceps strength and fall risk: a blinded, randomized trial of volunteers. Reg Anesth Pain Med 2013, 38(4):321-325.

15. Atkinson HD, Hamid I, Gupte CM, Russell RC, Handy JM: Postoperative fall after the use of the 3-in-1 femoral nerve block for knee surgery: a report of four cases. J Orthop Surg (Hong Kong) 2008, 16(3):381-384.

16. Feibel RJ, Dervin GF, Kim PR, Beaule PE: Major complications associated with femoral nerve catheters for knee arthroplasty: a word of caution. J Arthroplasty 2009, 24(6 Suppl):132-137.

17. Kandasami M, Kinninmonth AW, Sarungi M, Baines J, Scott NB: Femoral nerve block for total knee replacement - a word of caution. Knee 2009, 16(2):98-100.

18. Ilfeld BM, Duke KB, Donohue MC: The association between lower extremity continuous peripheral nerve blocks and patient falls after knee and hip arthroplasty. Anesth Analg 2010, 111(6):15521554.

19. Sharma S, lorio R, Specht LM, Davies-Lepie S, Healy WL: Complications of femoral nerve block for total knee arthroplasty. Clin Orthop Relat Res 2010, 468(1):135-140.

20. Wasserstein D, Farlinger C, Brull R, Mahomed N, Gandhi R: Advanced age, obesity and continuous femoral nerve blockade are independent risk factors for inpatient falls after primary total knee arthroplasty. J Arthroplasty 2013, 28(7):1121-1124. 
21. Pelt CE, Anderson AW, Anderson MB, Van Dine C, Peters CL: Postoperative falls after total knee arthroplasty in patients with a femoral nerve catheter: can we reduce the incidence? $\mathrm{J}$ Arthroplasty 2014, 29(6):1154-1157.

22. Turbitt LR, McHardy PG, Casanova M, Shapiro J, Li L, Choi S: Analysis of Inpatient Falls After Total Knee Arthroplasty in Patients With Continuous Femoral Nerve Block. Anesth Analg 2018, 127(1):224-227.

23. Nielsen KC, Klein SM, Steele SM: Femoral nerve blocks. Techniques in Regional Anesthesia and Pain Management 2003, 7(1):8-17.

24. Grevstad U, Mathiesen O, Valentiner LS, Jaeger P, Hilsted KL, Dahl JB: Effect of adductor canal block versus femoral nerve block on quadriceps strength, mobilization, and pain after total knee arthroplasty: a randomized, blinded study. Reg Anesth Pain Med 2015, 40(1):3-10.

25. Elkassabany NM, Antosh S, Ahmed M, Nelson C, Israelite C, Badiola I, Cai LF, Williams R, Hughes C, Mariano ER: The risk of falls after total knee arthroplasty with the use of a femoral nerve block versus an adductor canal block: a double-blinded randomized controlled study. Anesth Analg 2016, 122(5):1696-1703.

26. Li D, Tan Z, Kang P, Shen B, Pei F: Effects of multi-site infiltration analgesia on pain management and early rehabilitation compared with femoral nerve or adductor canal block for patients undergoing total knee arthroplasty: a prospective randomized controlled trial. Int Orthop 2017, 41(1):75-83.

27. Gao F, Ma J, Sun W, Guo W, Li Z, Wang W: Adductor Canal Block Versus Femoral Nerve Block for Analgesia After Total Knee Arthroplasty: A Systematic Review and Meta-analysis. Clin J Pain 2017, 33(4):356-368.

28. Koh IJ, Choi YJ, Kim MS, Koh HJ, Kang MS, In Y: Femoral nerve block versus adductor canal block for analgesia after total knee arthroplasty. Knee Surg Relat Res 2017, 29(2):87.

29. Ilfeld BM: Continuous Peripheral Nerve Blocks and Alternative Regional Analgesic Modalities: Clarification Regarding Relative Superiority. Anesth Analg 2017, 124(6):2088.

30. Lund J, Jenstrup MT, Jaeger P, Sorensen AM, Dahl JB: Continuous adductor-canal-blockade for adjuvant post-operative analgesia after major knee surgery: preliminary results. Acta Anaesthesiol Scand 2011, 55(1):14-19.

31. Shah NA, Jain NP: Is continuous adductor canal block better than continuous femoral nerve block after total knee arthroplasty? Effect on ambulation ability, early functional recovery and pain control: a randomized controlled trial. J Arthroplasty 2014, 29(11):2224-2229.

32. Kuang MJ, Xu LY, Ma JX, Wang Y, Zhao J, Lu B, Ma XL: Adductor canal block versus continuous femoral nerve block in primary total knee arthroplasty: A meta-analysis. Int J Surg 2016, 31:17-24.

33. Li D, Yang Z, Xie X, Zhao J, Kang P: Adductor canal block provides better performance after total knee arthroplasty compared with femoral nerve block: a systematic review and meta-analysis. Int Orthop 2016, 40(5):925-933.

34. Jiang X, Wang QQ, Wu CA, Tian W: Analgesic Efficacy of Adductor Canal Block in Total Knee Arthroplasty: A Meta-analysis and Systematic Review. Orthop Surg 2016, 8(3):294-300. 
35. Jæger P, Zaric D, Fomsgaard JS, Hilsted KL, Bjerregaard J, Gyrn J, Mathiesen O, Larsen TK, Dahl JB: Adductor Canal Block Versus Femoral Nerve Block for Analgesia After Total Knee Arthroplasty: A Randomized, Double-Blind Study. Reg Anesth Pain Med 2013, 38(6):526-532.

36. Kim DH, Lin Y, Goytizolo EA, Kahn RL, Maalouf DB, Manohar A, Patt ML, Goon AK, Lee YY, Ma Y et al: Adductor canal block versus femoral nerve block for total knee arthroplasty: a prospective, randomized, controlled trial. Anesthesiology 2014, 120(3):540-550.

37. Mudumbai SC, Kim TE, Howard SK, Workman JJ, Giori N, Woolson S, Ganaway T, King R, Mariano ER: Continuous adductor canal blocks are superior to continuous femoral nerve blocks in promoting early ambulation after TKA. Clin Orthop Relat Res 2014, 472(5):1377-1383.

38. Bolarinwa SA, Novicoff W, Cui Q: Reducing costly falls after total knee arthroplasty. World J Orthop 2018, 9(10):198.

39. Thacher RR, Hickernell TR, Grosso MJ, Shah R, Cooper HJ, Maniker R, Brown AR, Geller J: Decreased risk of knee buckling with adductor canal block versus femoral nerve block in total knee arthroplasty: a retrospective cohort study. Arthroplast Today 2017, 3(4):281-285.

40. Akai M, Doi T, Fujino K, Iwaya T, Kurosawa H, Nasu T: An outcome measure for Japanese people with knee osteoarthritis. J Rheumatol 2005, 32(8):1524-1532.

41. Ware JE, Jr., Sherbourne CD: The MOS 36-item short-form health survey (SF-36). I. Conceptual framework and item selection. Med Care 1992, 30(6):473-483.

42. Bellamy N, Buchanan WW, Goldsmith $\mathrm{CH}$, Campbell J, Stitt LW: Validation study of WOMAC: a health status instrument for measuring clinically important patient relevant outcomes to antirheumatic drug therapy in patients with osteoarthritis of the hip or knee. J Rheumatol 1988, 15(12):1833-1840.

43. Vaishya R, Vijay V, Demesugh DM, Agarwal AK: Surgical approaches for total knee arthroplasty. J Clin Orthop Trauma 2016, 7(2):71-79.

44. Mendell JR, Florence J: Manual muscle testing. Muscle Nerve 1990, 13 Suppl:S16-20.

45. Jenstrup MT, Jaeger P, Lund J, Fomsgaard JS, Bache S, Mathiesen O, Larsen TK, Dahl JB: Effects of adductor-canal-blockade on pain and ambulation after total knee arthroplasty: a randomized study. Acta Anaesthesiol Scand 2012, 56(3):357-364.

46. Gudmundsdottir S, Franklin JL: Continuous adductor canal block added to local infiltration analgesia (LIA) after total knee arthroplasty has no additional benefits on pain and ambulation on postoperative day 1 and 2 compared with LIA alone. Acta Orthop 2017, 88(5):537-542.

47. Canbek U, Akgun U, Aydogan NH, Kilinc CY, Uysal Al: Continuous adductor canal block following total knee arthroplasty provides a better analgesia compared to single shot: A prospective randomized controlled trial. Acta Orthop Traumatol Turc 2019, 53(5):334-339.

48. Kanda Y: Investigation of the freely available easy-to-use software 'EZR'for medical statistics. Bone Marrow Transplant 2013, 48(3):452-458.

49. Panella L, Tinelli C, Buizza A, Lombardi R, Gandolfı R: Towards objective evaluation of balance in the elderly: validity and reliability of a measurement instrument applied to the Tinetti test. Int $J$ Rehabil Res 2008, 31(1):65-72. 
50. Li D, Ma GG: Analgesic efficacy and quadriceps strength of adductor canal block versus femoral nerve block following total knee arthroplasty. Knee Surg Sports Traumatol Arthrosc 2016, 24(8):2614-2619.

51. Patterson ME, Bland KS, Thomas LC, Elliott CE, Soberon Jr JR, Nossaman BD, Osteen K: The adductor canal block provides effective analgesia similar to a femoral nerve block in patients undergoing total knee arthroplasty-a retrospective study. J Clin Anesth 2015, 27(1):39-44.

\section{Tables}

Table 1

Demographic data for FNB and ACB cohorts

\begin{tabular}{lccc}
\hline \multicolumn{1}{c}{ Demographics } & FNB & ACB & $\mathrm{p}$ \\
\hline Age, y & $75.7 \pm 6.2$ & $75.0 \pm 7.1$ & 0.63 \\
Male, n (\%) & $6(17)$ & $8(22)$ & 0.77 \\
\hline BMI, kg/m ${ }^{2}$ & $24.4 \pm 3.7$ & $26.0 \pm 2.8$ & 0.03 \\
\hline Dementia, n (\%) & $6(17)$ & $4(11)$ & 0.52 \\
\hline Cardiovascular disease, n (\%) & $12(33)$ & $16(43)$ & 0.47 \\
\hline Neurological disease, n (\%) & $7(19)$ & $6(16)$ & 0.768 \\
\hline Pulmonary disease, n (\%) & $4(11)$ & $9(24)$ & 0.221 \\
\hline Diabetes, n (\%) & $7(19)$ & $9(24)$ & 0.78 \\
\hline
\end{tabular}

Preoperative knee

\begin{tabular}{cccc}
\hline JKOM* I (pain) $^{*}$ & $63.1 \pm 25.4$ & $57.7 \pm 29.3$ & 0.45 \\
\hline JKOM* II to V (function) $^{*}$ & $49.9 \pm 19.2$ & $46.7 \pm 21.8$ & 0.536 \\
\hline Blood loss, $\mathrm{mL}$ & $271 \pm 128$ & $355 \pm 212$ & 0.045 \\
\hline
\end{tabular}

* Japanese Knee Osteoarthritis Measure:

JKOM I evaluates the pain component, with patients indicating their pain level on a continuous scale from 0-100, where 0 indicates "no pain at all" and 100 indicates "the most severe pain ever experienced." 
JKOM II to $\mathrm{V}$ are for knee function components. JKOM-II asks eight questions about knee pain and stiffness, JKOM-III asks ten questions about activities of daily living, JKOM-IV asks five questions about general activities that require leaving the house, and JKOM-V asks two questions about the patient's perception of their overall health and the contribution made by knee symptoms. Patients rate each of the 25 questions on a five-point Likert scale, with 0 indicating "best" and 4 indicating "worst". A total score of 0 indicates little loss of function, while a total score of 100 indicates severe loss of function.

Values were generated with two-sample two-tailed Student's $t$-test and Fisher's exact test. A $\mathrm{p}$ value $<0.05$ was considered significant.

Table 2

Comparison of days to first ambulation

\begin{tabular}{lcccc}
\hline & \multicolumn{4}{c}{ Initiation of ambulation: $\mathrm{n}(\%)$} \\
\cline { 2 - 5 } & POD 1 & POD 2 & POD 3 & POD 4 \\
\hline FNB $(\mathrm{n}=36)$ & $16(44.4)$ & $15(41.7)$ & $3(8.3)$ & $2(5.6)$ \\
ACB $(\mathrm{n}=37)$ & $30(81.1)$ & $5(13.5)$ & $1(2.7)$ & $1(2.7)$ \\
\hline
\end{tabular}

$(p=0.00575$, Fisher's exact test)

\section{Supplementary Files}

This is a list of supplementary files associated with this preprint. Click to download.

- TKAACBSupplementary4BMCsubmit.docx 